\title{
Approximation of the Stokes-Darcy system by optimization
}

\author{
V.J. Ervin* $\quad$ E.W. Jenkins ${ }^{\dagger} \quad$ Hyesuk Lee ${ }^{\ddagger}$
}

June 12, 2012

\begin{abstract}
A solution algorithm for the linear/nonlinear Stokes-Darcy coupled problem is proposed and investigated. The coupled system is formulated as a constrained optimal control problem, where a flow balance is forced across the interface, inflow, and outflow boundaries by minimizing a suitably defined functional. Optimization is achieved by exploiting a Neumann type boundary condition imposed on each subproblem as a control. A numerical algorithm is presented for a least squares functional whose solution yields a minimizer of the constrained optimization problem. Numerical experiments are provided to validate accuracy and efficiency of the algorithm.
\end{abstract}

Key words Stokes-Darcy, least squares, finite element method

AMS subject classifications. 65N55, 65N30, 76D07, 76M10

\section{Introduction}

The Stokes-Darcy coupled system has received a good deal of attention in the area of scientific computing due to its many important applications in modeling groundwater flow, filtration processes, petroleum reservoirs, etc. [1, 2, 5, 6, 8, 10, 18, 21]. A variety of solution algorithms have been proposed for solving the Stokes-Darcy system (see, for instance, [5] and references therein). Fully coupled approaches are considered in $[1,2,21,10,24]$, while decoupling strategies are analyzed in $[3,5,9,13,11,25]$. Of the fully coupled approaches, some introduce new finite element spaces $[1,2]$ (along with modified solution algorithms), and others either introduce Lagrange multiplier spaces $[21,10]$ or fully discontinuous approximations [24] to resolve the coupled system.

The decoupling strategies employ several domain decomposition techniques to allow use of optimized algorithms for the Stokes and Darcy subproblems. The mortar space methods introduce a subproblem based on mass conservation on the interface [24, 11, 3, 12], while the Robin-Robin domain decomposition methods [5,9] combine the conservation of mass

\footnotetext{
${ }^{*}$ Department of Mathematical Sciences, Clemson University, Clemson, SC 29634-0975 (vjervin@clemson.edu).

${ }^{\dagger}$ Department of Mathematical Sciences, Clemson University, Clemson, SC 29634-0975 (lea@clemson.edu).

${ }^{\ddagger}$ Department of Mathematical Sciences, Clemson University, Clemson, SC 29634-0975 (hklee@mail.clemson.edu), Partially supported by the NSF under grant no. DMS-1016182.
} 
and balance of normal forces on the interface into Robin conditions associated with each subproblem. A two grid solution approach was proposed and analyzed in [4], with an initial coupled approximation computed on a coarse mesh and then a correction, computable in parallel, determined on a fine mesh for the Stokes and Darcy subproblems.

The solution algorithm considered in this paper is based on an optimization approach. The optimization-based domain decomposition was initially studied in [17] for the Poisson problem and later extended to the Navier-Stokes and the Boussinesq equations [16, 22]. There, the minimized functional measures the jump in the dependent variables across the common boundary between subdomains. The dependent variables were solutions of subproblems with a suitably chosen boundary condition on the interface as a control. The jump of subproblem solutions along the interfaces between subdomains was measured in the $L^{2}$ norm. This is mathematically reasonable as the traces of subproblem solutions on the interface were $L^{2}$ integrable. However, this is not the case for the Stokes-Darcy system. Thus, the functional to be minimized should be carefully designed so that its definition is consistent with the chosen function spaces for the Stokes and Darcy problems, respectively. More detailed discussion will be given in Section 3, where the optimization problem is introduced.

For the modeling equations, the boundary conditions we consider on the inflow and outflow boundaries are specified flow rates. Such boundary conditions are called defective boundary conditions because they do not determine a unique solution to the modeling equations. Similar to the domain decomposition method described above, the defective boundary condition can be treated by flow matching, i.e., by minimizing the difference between given rates and computed values [23]. Again, the control for optimization is Neumann conditions on inflow and outflow boundaries, respectively.

To formulate an optimal control problem for the Stokes-Darcy system with defective boundary conditions we introduce a dual-objective functional including two main objective terms; one for the continuity of the normal components of the Stokes and Darcy velocities and the other for flow matching. Constant weights are introduced to balance the objectives, including a penalty term.

This paper is organized as follows. In the next section we describe the model problem, introduce function spaces and state an existence result. In Section 3 the constrained minimization problem is described and the existence of an optimal solution is shown. In Section 4, we reformulate the optimization problem as a least squares problem and present a computational algorithm. We then extend the method to the nonlinear Stokes-Darcy system in Section 5. Numerical experiments are given in Section 6 and some concluding remarks concerning the method and its efficiency are provided in Section 7.

\section{Model equations}

Let $\Omega_{f}, \Omega_{p}$ be bounded fluid and porous media domains in $\mathbb{R}^{d}$, respectively, and $I$ denote the interface between two domains. Let $\Gamma_{i n}, \Gamma_{\text {out }}$ be inflow and outflow boundaries such that $\Gamma_{\text {in }} \subset \partial \Omega_{f} \backslash I, \Gamma_{\text {out }} \subset \partial \Omega_{p} \backslash I$ and define $\Gamma_{f}:=\partial \Omega_{f} \backslash\left(I \cup \Gamma_{\text {in }}\right), \Gamma_{p}:=\partial \Omega_{p} \backslash\left(I \cup \Gamma_{\text {out }}\right)$, as illustrated in Figure 2.1. 


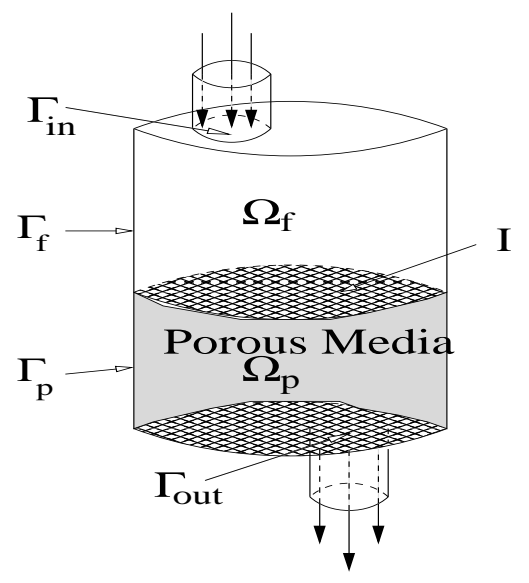

Figure 2.1: Illustration of flow domain.

Consider the Stokes and Darcy problems:

$$
\begin{aligned}
-2 \nu_{f} \nabla \cdot D\left(\mathbf{u}_{f}\right)+\nabla p_{f} & =\mathbf{f}_{f} \quad \text { in } \Omega_{f}, \\
\nabla \cdot \mathbf{u}_{f} & =0 \quad \text { in } \Omega_{f}, \\
\mathbf{u}_{f} & =\mathbf{0} \quad \text { on } \Gamma_{f}, \\
-\int_{\Gamma_{\text {in }}} \mathbf{u}_{f} \cdot \mathbf{n}_{f} d \Gamma_{f} & =Q_{1}, \\
\mu_{e f f} \mathbf{K}^{-1} \mathbf{u}_{p}+\nabla p_{p} & =\mathbf{0} \quad \text { in } \Omega_{p} \\
\nabla \cdot \mathbf{u}_{p} & =f_{p} \quad \text { in } \Omega_{p}, \\
\mathbf{u}_{p} \cdot \mathbf{n}_{p} & =0 \quad \text { on } \Gamma_{p} \\
\int_{\Gamma_{\text {out }}} \mathbf{u}_{p} \cdot \mathbf{n}_{p} d \Gamma_{p} & =Q_{2}
\end{aligned}
$$

where $\mathbf{u}_{f}$ denotes the fluid velocity, $p_{f}$ the fluid pressure, $\mathbf{u}_{p}$ the Darcy velocity and $p_{p}$ the Darcy pressure. In $(2.1), D(\mathbf{v}):=\left(\nabla \mathbf{v}+\nabla \mathbf{v}^{T}\right) / 2$ is the rate of the strain tensor, $\nu_{f}$ the fluid viscosity and $\mathbf{f}_{f}$ the body force. The term $f_{p}$ in (2.6) models any sink/source terms in $\Omega_{p}$. In (2.5) $\mu_{\text {eff }}$ represents an effective fluid viscosity, and $\mathbf{K}$ the permeability (symmetric, positive definite) tensor of the porous media. For simplicity, we take $\nu_{p} \mathbf{I}=\mu_{\text {eff }} \mathbf{K}^{-1}$. In (2.4), (2.8) $\mathbf{n}_{f}, \mathbf{n}_{p}$ denote outward unit normal vectors associated with $\Omega_{f}, \Omega_{p}$, respectively. Note that on the inflow and outflows boundaries only the fluid flow rates are specified. Such boundary conditions are known as defective boundary conditions [19]. By the mass conservation law the specified flow rates satisfy $Q_{1}+\int_{\Omega_{p}} f_{p} d \Omega_{p}=Q_{2}$. On the interface $I$, the following conditions are imposed

$$
\begin{aligned}
\mathbf{u}_{f} \cdot \mathbf{n}_{f}+\mathbf{u}_{p} \cdot \mathbf{n}_{p} & =0 \\
\mathbf{n}_{f} \cdot\left(p_{f}-2 \nu_{f} D\left(\mathbf{u}_{f}\right)\right) \cdot \mathbf{n}_{f} & =p_{p} \\
\mathbf{n}_{f} \cdot\left(p_{f}-2 \nu_{f} D\left(\mathbf{u}_{f}\right)\right) \cdot \mathbf{t}_{j} & =\alpha \mathbf{u}_{f} \cdot \mathbf{t}_{j}, j=1, \ldots, d-1,
\end{aligned}
$$


where $\mathbf{t}_{j}, j=1, \ldots, d-1$, denote the (orthogonal) unit tangent unit vector on $I$. The first two interface conditions enforce continuity of the normal component of velocities and continuity of the normal component of the normal stress tensor on the interface, and the third is the Beavers-Joseph-Saffmann condition [20].

In this paper we develop a numerical algorithm for the approximation of the coupled system based on a minimization technique. The problem is formulated as a least squares problem where the conditions (2.4), (2.8), (2.9) are satisfied by minimization, and the condition (2.10) is naturally imposed by introducing a common Neumann type condition for the Stokes and Darcy subproblems.

For the mathematical formulation we use the Sobolev spaces $W^{m, p}(\Theta)$, with norms $\|\cdot\|_{m, p, \Theta}$. In the case $p=2$, the Sobolev space $W^{m, 2}(\Theta)$ is denoted by $H^{m}(\Theta)$ with the norm $\|\cdot\|_{m, \Theta}$. The corresponding space of vector valued or tensor-valued functions is denoted by a boldface font. By $(\cdot, \cdot)_{\Theta}$ we denote the $L^{2}$ inner product over $\Theta$.

If $\Theta=\Omega_{f}$ or $\Omega_{p}$, and the context is clear, $\Theta$ is omitted, i.e., $(\cdot, \cdot)=(\cdot, \cdot)_{\Omega_{f}}$ or $(\cdot, \cdot) \Omega_{p}$ for functions defined in $\Omega_{f}$ and $\Omega_{p}$. For $\gamma \subset \partial \Omega_{f} \cup \partial \Omega_{p}$, we use $\langle\cdot, \cdot\rangle_{\gamma}$ to denote the duality pairing between $H^{-1 / 2}(\gamma)$ and $H^{1 / 2}(\gamma)$.

The function spaces for the velocities $\mathbf{u}_{f}, \mathbf{u}_{p}$ and the pressures $p_{f}, p_{p}$ are defined by:

$$
\begin{aligned}
& \mathbf{X}_{f}:=\left\{\mathbf{v} \in \mathbf{H}^{1}\left(\Omega_{f}\right): \mathbf{v}=\mathbf{0} \quad \text { on } \Gamma_{f}\right\} \\
& Q_{f}:=L^{2}\left(\Omega_{f}\right), \\
& \mathbf{X}_{p}:=\mathbf{H}^{\operatorname{div}}\left(\Omega_{p}\right)=\left\{\mathbf{v} \in \mathbf{L}^{2}\left(\Omega_{p}\right): \nabla \cdot \mathbf{v} \in \mathbf{L}^{2}\left(\Omega_{p}\right), \mathbf{v} \cdot \mathbf{n}_{p}=0 \quad \text { on } \Gamma_{p}\right\}, \\
& Q_{p}:=L^{2}\left(\Omega_{p}\right),
\end{aligned}
$$

where the Darcy velocity space $\mathbf{X}_{p}$ is equipped with the norm

$$
\left\|\mathbf{v}_{p}\right\|_{\mathbf{H}^{d i v}\left(\Omega_{p}\right)}:=\left(\left\|\mathbf{v}_{p}\right\|_{0, \Omega_{p}}^{2}+\left\|\nabla \cdot \mathbf{v}_{p}\right\|_{0, \Omega_{p}}^{2}\right)^{1 / 2}
$$

Introduce

$$
\begin{aligned}
\mathbf{S} & :=H^{1 / 2}(I) \times \mathbf{H}^{-1 / 2}\left(\Gamma_{\text {in }}\right) \times H^{1 / 2}\left(\Gamma_{\text {out }}\right) \\
\mathbf{g} & :=\left[g_{1}, \mathbf{g}_{2}, g_{3}\right]^{T} \in \mathbf{S}, \\
\|\mathbf{g}\|_{\mathbf{S}} & :=\left(\left\|g_{1}\right\|_{H^{1 / 2}(I)}^{2}+\left\|\mathbf{g}_{2}\right\|_{\mathbf{H}^{-1 / 2}\left(\Gamma_{\text {in }}\right)}^{2}+\left\|g_{3}\right\|_{H^{1 / 2}\left(\Gamma_{\text {out }}\right)}^{2}\right)^{1 / 2}
\end{aligned}
$$

and consider the following variational formulation for the Stokes and Darcy problems. Given $\mathbf{g} \in \mathbf{S}$ determine $\left(\mathbf{u}_{f}, p_{f}\right)$ and $\left(\mathbf{u}_{p}, p_{p}\right)$ satisfying

$$
\begin{aligned}
& 2 \nu_{f}\left(D\left(\mathbf{u}_{f}\right), D\left(\mathbf{v}_{f}\right)\right)-\left(p_{f}, \nabla \cdot \mathbf{v}_{f}\right)+\alpha\left(\mathbf{u}_{f} \cdot \mathbf{t}, \mathbf{v}_{f} \cdot \mathbf{t}\right)_{I} \\
& \quad=\left(\mathbf{f}, \mathbf{v}_{f}\right)+\left\langle g_{1}, \mathbf{v}_{f} \cdot \mathbf{n}_{f}\right\rangle_{I}+\left\langle\mathbf{g}_{2}, \mathbf{v}_{f}\right\rangle_{\Gamma_{\text {in }}} \quad \forall \mathbf{v}_{f} \in \mathbf{X}_{f}, \\
& \left(q_{f}, \nabla \cdot \mathbf{u}_{f}\right)=0 \quad \forall q_{f} \in Q_{f}, \\
& \nu_{p}\left(\mathbf{u}_{p}, \mathbf{v}_{p}\right)-\left(p_{p}, \nabla \cdot \mathbf{v}_{p}\right)=\left\langle g_{1}, \mathbf{v}_{p} \cdot \mathbf{n}_{p}\right\rangle_{I}+\left\langle g_{3}, \mathbf{v}_{p} \cdot \mathbf{n}_{p}\right\rangle_{\Gamma_{\text {out }}} \quad \forall \mathbf{v}_{p} \in \mathbf{X}_{p}, \\
& \left(q_{p}, \nabla \cdot \mathbf{u}_{p}\right)=\left(f_{p}, q_{p}\right) \quad \forall q_{p} \in Q_{p} .
\end{aligned}
$$


For $\mathbf{u} \in \mathbf{H}^{\text {div }}\left(\Omega_{p}\right), \mathbf{u} \cdot \mathbf{n}_{p} \in H^{-1 / 2}\left(\partial \Omega_{p}\right)$. For $p \in H^{1 / 2}(I)$, duality pairing does not define $\left\langle\mathbf{u} \cdot \mathbf{n}_{p}, p\right\rangle_{I}$, as $\mathbf{u} \cdot \mathbf{n}_{p}$ acts on functions in $H^{1 / 2}\left(\partial \Omega_{p}\right)$. Following the work of Galvis and Sarkis ([12], Lemma 2.1), given $\Gamma_{s} \subset \partial \Omega_{p}, r \in H^{1 / 2}\left(\Gamma_{s}\right)$, let $E_{\Gamma_{s}}^{1 / 2} r \in H^{1 / 2}\left(\partial \Omega_{p}\right)$ denote the extension of $r$ to $\partial \Omega_{p}$. Then, for $f \in H^{-1 / 2}\left(\partial \Omega_{p}\right)$, we denote

$$
\langle f, r\rangle_{\Gamma_{s}}:=\left\langle f, E_{\Gamma_{s}}^{1 / 2} r\right\rangle_{\partial \Omega_{p}} .
$$

Also, as given in [12], for $f \in H^{-1 / 2}\left(\partial \Omega_{p}\right),\left.f\right|_{\Gamma_{s}}=0$ is defined as

$$
\left\langle f, E_{00, \Gamma_{s}}^{1 / 2} w\right\rangle_{\partial \Omega_{p}}=0, \text { for all } w \in H_{00}^{1 / 2}\left(\Gamma_{s}\right),
$$

where $E_{00, \Gamma_{s}}^{1 / 2} w$ denotes the extension by 0 of $w$ to $\partial \Omega_{p} \backslash \Gamma_{s}$.

Using integration by parts and (2.10), it can be easily seen that $\mathbf{g}$ plays a role as

$$
\begin{aligned}
& g_{1}=\left.\left(\mathbf{n}_{f} \cdot 2 \nu_{f} D\left(\mathbf{u}_{f}\right) \cdot \mathbf{n}_{f}-p_{f}\right)\right|_{I}, \\
& \mathbf{g}_{2}=\left.\left(\mathbf{n}_{f} \cdot 2 \nu_{f} D\left(\mathbf{u}_{f}\right)-p_{f} \mathbf{n}_{f}\right)\right|_{\Gamma_{\text {in }}}, \\
& g_{3}=-\left.p_{p}\right|_{\Gamma_{\text {out }}},
\end{aligned}
$$

and the boundary condition (2.10) is weakly imposed through $g_{1}$ on the right hand side of (2.15) and (2.17).

Remark 2.1 The coupled Stokes-Darcy problem is analyzed in [5, 10, 21], where a Lagrange multiplier $\lambda$ is introduced to impose the interface condition (2.10).

For the existence, uniqueness, and boundedness of the solutions to (2.15)-(2.16) and (2.17)-(2.18), from [11] we have the following.

Lemma 2.2 Given $\mathbf{f}_{f} \in \mathbf{X}_{f}^{-1}, f_{p} \in L^{2}\left(\Omega_{p}\right), \mathbf{g} \in \mathbf{S}$, the Stokes and Darcy systems (2.15)(2.16) and (2.17)-(2.18) have a unique solution $\left(\mathbf{u}_{f}, p_{f}\right),\left(\mathbf{u}_{p}, p_{p}\right)$, respectively, satisfying

$$
\begin{gathered}
\left\|\mathbf{u}_{f}\right\|_{1, \Omega_{f}}+\left\|p_{f}\right\|_{0, \Omega_{f}} \leq C\left(\|\mathbf{f}\|_{-1, \Omega_{f}}+\left\|g_{1}\right\|_{H^{1 / 2}(I)}+\left\|\mathbf{g}_{2}\right\|_{\mathbf{H}^{-1 / 2}\left(\Gamma_{\text {in }}\right)}\right) \\
\left\|\mathbf{u}_{p}\right\|_{\mathbf{H}^{d i v}\left(\Omega_{p}\right)}+\left\|p_{p}\right\|_{0, \Omega_{p}} \leq C\left(\left\|f_{p}\right\|_{0, \Omega_{p}}+\left\|g_{1}\right\|_{H^{1 / 2}(I)}+\left\|g_{3}\right\|_{H^{1 / 2}\left(\Gamma_{\text {out }}\right)}\right) .
\end{gathered}
$$

\section{Optimal control problem}

To formulate an optimization problem let the interface boundary $I$ be partitioned into nonoverlapped segments $I_{i}$ for $i=1,2, \ldots, n$ such that $I=\cup_{i=1}^{n} I_{i}$. In order to satisfy the boundary conditions $(2.4),(2.8),(2.9)$, we wish to find a function $\mathbf{g} \in \mathbf{S}$ such that $\left(\mathbf{u}_{f}, p_{f}\right)$, $\left(\mathbf{u}_{p}, p_{p}\right)$, solutions of (2.15)-(2.16) and (2.17)-(2.18), respectively, minimize

$$
\begin{aligned}
& \mathcal{J}(\mathbf{g}):=\frac{1}{2}\left(\omega_{1} \sum_{i=1}^{n}\left(\frac{1}{\sqrt{\left|I_{i}\right|}} \int_{I_{i}} \mathbf{u}_{f} \cdot \mathbf{n}_{f}+\mathbf{u}_{p} \cdot \mathbf{n}_{p} d I_{i}\right)^{2}+\delta\|\mathbf{g}\|_{\mathbf{S}}^{2} d I\right. \\
& \left.+\omega_{2}\left(\frac{1}{\sqrt{\left|\Gamma_{\text {in }}\right|}} \int_{\Gamma_{\text {in }}} \mathbf{u}_{f} \cdot \mathbf{n}_{f} d \Gamma_{\text {in }}+\frac{Q_{1}}{\sqrt{\left|\Gamma_{\text {in }}\right|}}\right)^{2}+\omega_{2}\left(\frac{1}{\sqrt{\left|\Gamma_{\text {out }}\right|}}\left\langle\mathbf{u}_{p} \cdot \mathbf{n}_{p}, 1\right\rangle_{\Gamma_{\text {out }}}-\frac{Q_{2}}{\sqrt{\left|\Gamma_{\text {out }}\right|}}\right)^{2}\right),
\end{aligned}
$$


where $|\gamma|:=\operatorname{meas}(\gamma)$ for $\gamma \subset \partial \Omega_{f} \cup \partial \Omega_{p}$. In (3.1) $\delta>0$ is a penalty parameter and $\omega_{1}, \omega_{2}>0$ are weights such that $\omega_{1}+\omega_{2}=1$.

Minimizing the first term of (3.1) seeks to weakly impose the interface condition (2.9) by forcing flow balance across the interface segments $I_{i}$. Physically this is appropriate as $\mathbf{u}_{f}$ and $\mathbf{u}_{p}$ denote different quantities, $\mathbf{u}_{p}$ representing an averaged fluid velocity. Note that, assuming $\mathbf{u}_{f} \cdot \mathbf{n}_{f}+\mathbf{u}_{p} \cdot \mathbf{n}_{p}$ is continuous,

$$
\begin{aligned}
& \lim _{n \rightarrow \infty} \sum_{i=1}^{n}\left(\frac{1}{\sqrt{\left|I_{i}\right|}} \int_{I_{i}} \mathbf{u}_{f} \cdot \mathbf{n}_{f}+\mathbf{u}_{p} \cdot \mathbf{n}_{p} d I_{i}\right)^{2} \\
& =\lim _{n \rightarrow \infty} \sum_{i=1}^{n}\left|I_{i}\right|\left(\left.\left(\mathbf{u}_{f} \cdot \mathbf{n}_{f}+\mathbf{u}_{p} \cdot \mathbf{n}_{p}\right)\right|_{\xi_{i}}\right)^{2}, \text { for some } \xi_{i} \in I_{i} \\
& =\int_{I}\left(\mathbf{u}_{f} \cdot \mathbf{n}_{f}+\mathbf{u}_{p} \cdot \mathbf{n}_{p}\right)^{2} d I
\end{aligned}
$$

So, as $n \rightarrow \infty, \quad$ for $\left(\mathbf{u}_{f} \cdot \mathbf{n}_{f}+\mathbf{u}_{p} \cdot \mathbf{n}_{p}\right)$ continuous on $I$, forcing $\sum_{i=1}^{n}\left(\frac{1}{\sqrt{\left|I_{i}\right|}} \int_{I_{i}} \mathbf{u}_{f} \cdot \mathbf{n}_{f}+\mathbf{u}_{p} \cdot \mathbf{n}_{p} d I_{i}\right)^{2}=0$ imposes (2.9) pointwise on $I$. However, for the general setting $\mathbf{u}_{p} \in \mathbf{H}^{\operatorname{div}}\left(\Omega_{p}\right), \mathbf{u}_{p} \cdot \mathbf{n}_{p} \in H^{-1 / 2}\left(\partial \Omega_{p}\right)$, the integral in (3.2) is not defined, and we interpret $\int_{I_{i}} \mathbf{u}_{p} \cdot \mathbf{n}_{p} d I_{i}=\left\langle\mathbf{u}_{p} \cdot \mathbf{n}_{p}, E_{I_{i}}^{1 / 2} 1\right\rangle_{\partial \Omega_{p}}$.

In (3.1) the factors $1 / \sqrt{\left|\Gamma_{\text {in }}\right|}$ and $1 / \sqrt{\left|\Gamma_{\text {out }}\right|}$ are used as normalizing constants to account for possible different lengths of the inflow and outflow boundaries.

Define the admissibility set $\mathcal{U}_{a d}$ as

$$
\mathcal{U}_{a d}:=\left\{\left(\mathbf{u}_{f}, p_{f}, \mathbf{u}_{p}, p_{p}, \mathbf{g}\right) \in \mathbf{X}_{f} \times Q_{f} \times \mathbf{X}_{p} \times Q_{p} \times \mathbf{S}: \mathcal{J}\left(\mathbf{u}_{f}, p_{f}, \mathbf{u}_{p}, p_{p}, \mathbf{g}\right)<\infty\right\} .
$$

We formulate the optimal control in the following terms:

$$
\begin{aligned}
& \text { Find }\left(\mathbf{u}_{f}, p_{f}, \mathbf{u}_{p}, p_{p}, \mathbf{g}\right) \in \mathcal{U}_{a d} \text { such that the functional (3.1) is minimized } \\
& \text { subject to }(2.15)-(2.18) .
\end{aligned}
$$

As $Q_{2}=Q_{1}+\int_{\Omega_{p}} f_{p} d \Omega_{p}$, due to the mass conservation law, $g_{3}$ in $\mathbf{g}$ may be set to an arbitrary function, say 0 , and an optimal $\mathbf{g}=\left(g_{1}, \mathbf{g}_{2}, 0\right)$ may be sought to minimize the functional (3.1). Using both $\mathbf{g}_{2}$ and $g_{3}$ as controls for the defective boundary conditions on $\Gamma_{\text {in }}$ and $\Gamma_{\text {out }}$ may result in an over controlled system. This case will be discussed in Section 6. However, for generality, we will assume a control function of the form $\mathbf{g}=\left(g_{1}, \mathbf{g}_{2}, g_{3}\right)$.

To establish the existence of an optimal solution of (3.4) we firstly show that $\mathcal{J}(\mathbf{g})$ is a continuous function of $\mathbf{g}$.

Lemma 3.1 The functional $\mathcal{J}(\cdot): \mathbf{S} \rightarrow \mathbb{R}$ is continuous.

Proof: From the bounds for $\mathbf{u}_{f}$ and $\mathbf{u}_{p}$ given in (2.22) and (2.23), respectively, we have that $\mathbf{u}_{f}$ and $\mathbf{u}_{p}$ are continuous functions of $\mathbf{g}$. Thus it suffices to show that for $\Gamma_{1} \subset \partial \Omega_{f}$, $\Gamma_{2} \subset \Omega_{p}, \int_{\Gamma_{1}} \mathbf{u}_{f} \cdot \mathbf{n}_{f} d \Gamma_{1}$ and $\left\langle\mathbf{u}_{p} \cdot \mathbf{n}_{p}, 1\right\rangle_{\Gamma_{2}}$ are continuous functions of $\mathbf{u}_{f}$ and $\mathbf{u}_{p}$, respectively. 
For the integral over $\Gamma_{1}$, using the Trace Theorem,

$$
\int_{\Gamma_{1}} \mathbf{u}_{f} \cdot \mathbf{n}_{f} d \Gamma_{1} \leq\left|\Gamma_{1}\right|^{1 / 2}\left\|\mathbf{u}_{f} \cdot \mathbf{n}_{f}\right\|_{L^{2}\left(\Gamma_{1}\right)} \leq C\left|\Gamma_{1}\right|^{1 / 2}\left\|\mathbf{u}_{f}\right\|_{1, \Omega_{f}}
$$

For the $\Gamma_{2}$ term, using the definition given above,

$$
\begin{aligned}
\left\langle\mathbf{u}_{p} \cdot \mathbf{n}_{p}, 1\right\rangle_{\Gamma_{2}}=\left\langle\mathbf{u}_{p} \cdot \mathbf{n}_{p}, E_{\Gamma_{2}}^{1 / 2} 1\right\rangle_{\partial \Omega_{p}} & \leq\left\|\mathbf{u}_{p} \cdot \mathbf{n}_{p}\right\|_{H^{-1 / 2}\left(\partial \Omega_{p}\right)}\left\|E_{\Gamma_{2}}^{1 / 2} 1\right\|_{H^{1 / 2}\left(\partial \Omega_{p}\right)} \\
& \leq C\left\|\mathbf{u}_{p}\right\|_{\mathbf{H}^{d i v}\left(\Omega_{p}\right)}\left|\Gamma_{2}\right|^{1 / 2} .
\end{aligned}
$$

Theorem 3.2 There exists a solution $\left(\mathbf{u}_{f}, p_{f}, \mathbf{u}_{p}, p_{p}, \mathbf{g}\right) \in \mathbf{X}_{f} \times Q_{f} \times \mathbf{X}_{p} \times Q_{p} \times \mathbf{S}$ of the optimal control problem (3.4).

Proof: In this proof we use the notation $\left(\mathbf{u}_{f}(\mathbf{g}), p_{f}(\mathbf{g})\right),\left(\mathbf{u}_{p}(\mathbf{g}), p_{p}(\mathbf{g})\right)$ to denote the solutions of (2.15)-(2.16), and (2.17)-(2.18), respectively, for the given data $\mathbf{g}$. We first note that the admissible set $\mathcal{U}_{a d}$ is clearly not empty, e.g., $\left(\mathbf{u}_{f}(\mathbf{0}), p_{f}(\mathbf{0}), \mathbf{u}_{p}(\mathbf{0}), p_{p}(\mathbf{0}), \mathbf{0}\right) \in$ $\mathcal{U}_{a d}$. Let $\mathbf{g}^{m}$ be a minimizing sequence for the optimal control problem and set $\mathbf{u}_{f}^{m}=$ $\mathbf{u}_{f}\left(\mathbf{g}^{m}\right), p_{f}^{m}=p_{f}\left(\mathbf{g}^{m}\right), \mathbf{u}_{p}^{m}=\mathbf{u}_{p}\left(\mathbf{g}^{m}\right), p_{p}^{m}=p_{p}\left(\mathbf{g}^{m}\right)$. Then $\left(\mathbf{u}_{f}^{m}, p_{f}^{m}, \mathbf{u}_{p}^{m}, p_{p}^{m}, \mathbf{g}^{m}\right) \in \mathcal{U}_{a d}$ for all $m$, and satisfies

$$
\lim _{m \rightarrow \infty} \mathcal{J}\left(\mathbf{u}_{f}^{m}, p_{f}^{m}, \mathbf{u}_{p}^{m}, p_{p}^{m}, \mathbf{g}^{m}\right)=\inf _{\left(\mathbf{u}_{f}, p_{f}, \mathbf{u}_{p}, p_{p}, \mathbf{g}\right) \in \mathcal{U}_{a d}} \mathcal{J}\left(\mathbf{u}_{f}, p_{f}, \mathbf{u}_{p}, p_{p}, \mathbf{g}\right) .
$$

By the definition of $\mathcal{U}_{a d}$, we have

$$
\begin{aligned}
& 2 \nu_{f}\left(D\left(\mathbf{u}_{f}^{m}\right), D\left(\mathbf{v}_{f}\right)\right)-\left(p_{f}^{m}, \nabla \cdot \mathbf{v}_{f}\right)+\alpha\left(\mathbf{u}_{f}^{m} \cdot \mathbf{t}, \mathbf{v}_{f} \cdot \mathbf{t}\right)_{I} \\
& =\left(\mathbf{f}, \mathbf{v}_{f}\right)+\left\langle g_{1}^{m}, \mathbf{v}_{f} \cdot \mathbf{n}_{f}\right\rangle_{I}+\left\langle\mathbf{g}_{2}^{m}, \mathbf{v}_{f}\right\rangle_{\Gamma_{i n}} \quad \forall \mathbf{v}_{f} \in \mathbf{X}_{f}, \\
& \left(q_{f}, \nabla \cdot \mathbf{u}_{f}^{m}\right)=0 \quad \forall q_{f} \in Q_{f}(\Omega), \\
& \nu_{p}\left(\mathbf{u}_{p}^{m}, \mathbf{v}_{p}\right)-\left(p_{p}^{m}, \nabla \cdot \mathbf{v}_{p}\right)=\left\langle g_{1}^{m}, \mathbf{v}_{p} \cdot \mathbf{n}_{p}\right\rangle_{I}+\left\langle g_{3}^{m}, \mathbf{v}_{p} \cdot \mathbf{n}_{p}\right\rangle_{\Gamma_{\text {out }}} \quad \forall \mathbf{v}_{p} \in \mathbf{X}_{p}, \\
& \left(q_{p}, \nabla \cdot \mathbf{u}_{p}^{m}\right)=\left(f_{p}, q_{p}\right) \quad \forall q_{p} \in Q_{p}(\Omega) .
\end{aligned}
$$

The sequence $\mathbf{g}^{m}$ is uniformly bounded in $\mathbf{S}$ from (3.3), and the corresponding sequence $\left(\mathbf{u}_{f}^{m}\left(\mathbf{g}^{m}\right), p_{f}^{m}\left(\mathbf{g}^{m}\right), \mathbf{u}_{p}^{m}\left(\mathbf{g}^{m}\right), p_{p}^{m}\left(\mathbf{g}^{m}\right)\right)$ is uniformly bounded in $\mathbf{X}_{f} \times Q_{f} \times \mathbf{X}_{p} \times Q_{p}$ from (2.22)-(2.23). We can then extract subsequences, still denoted by $\left(\mathbf{u}_{f}^{m}, p_{f}^{m}, \mathbf{u}_{p}^{m}, p_{p}^{m}, \mathbf{g}^{m}\right)$, such that

$$
\left.\begin{array}{ccc}
\mathbf{g}^{m} \rightarrow \tilde{\mathbf{g}} & \text { in } & \mathbf{S}, \\
p_{f}^{m} \rightarrow \tilde{p}_{f} & \text { in } & Q_{f}, \\
\mathbf{u}_{f}^{m} \rightarrow \tilde{\mathbf{u}}_{f} & \text { in } & \mathbf{X}_{f}, \\
\mathbf{u}_{f}^{m} \rightarrow \tilde{\mathbf{u}}_{f} & \text { in } & \mathbf{L}^{2}\left(\Omega_{f}\right), \\
p_{p}^{m} \rightarrow \tilde{p}_{p} & \text { in } & Q_{p}, \\
\mathbf{u}_{p}^{m} \rightarrow \tilde{\mathbf{u}}_{p} & \text { in } & \mathbf{X}_{p}, \\
\mathbf{u}_{p}^{m} \rightarrow \tilde{\mathbf{u}}_{p} & \text { in } & \mathbf{L}^{2}\left(\Omega_{p}\right)
\end{array}\right\}
$$


for some $\left(\tilde{\mathbf{u}}_{f}, \tilde{p}_{f}, \tilde{\mathbf{u}}_{p}, \tilde{p}_{p}, \tilde{\mathbf{g}}\right) \in \mathbf{X}_{f} \times Q_{f} \times \mathbf{X}_{p} \times Q_{p} \times \mathbf{S}$. Using (3.11), we can pass to the limit in (2.15)-(2.18) to determine that $\left(\tilde{\mathbf{u}}_{f}, \tilde{p}_{f}, \tilde{\mathbf{u}}_{p}, \tilde{p}_{p}, \tilde{\mathbf{g}}_{)}\right)$satisfies (2.15)-(2.18). Now, by the continuity of $\mathcal{J}(\cdot, \cdot, \cdot, \cdot, \cdot)$, we conclude that $\left(\tilde{\mathbf{u}}_{f}, \tilde{p}_{f}, \tilde{\mathbf{u}}_{p}, \tilde{p}_{p}, \tilde{\mathbf{g}}\right)$ is an optimal solution i.e.,

$$
\inf _{\left(\mathbf{u}_{f}, p_{f}, \mathbf{u}_{p}, p_{p}, \mathbf{g}\right) \in \mathcal{U}_{a d}} \mathcal{J}\left(\mathbf{u}_{f}, p_{f}, \mathbf{u}_{p}, p_{p}, \mathbf{g}\right)=\lim _{m \rightarrow \infty} \mathcal{J}\left(\mathbf{u}_{f}^{m}, p_{f}^{m}, \mathbf{u}_{p}^{m}, p_{p}^{m}, \mathbf{g}^{m}\right)=\mathcal{J}\left(\tilde{\mathbf{u}}_{f}, \tilde{p}_{f}, \tilde{\mathbf{u}}_{p}, \tilde{p}_{p}, \tilde{\mathbf{g}}\right) .
$$

Thus, an optimal solution to (3.4) exists.

\section{Least squares approach}

In order to develop a computational algorithm we formulate the optimization problem (3.4) as a least squares problem.

Define the linear operator $N: \mathbf{S} \rightarrow R^{n} \times R^{2} \times \mathbf{S}$ by

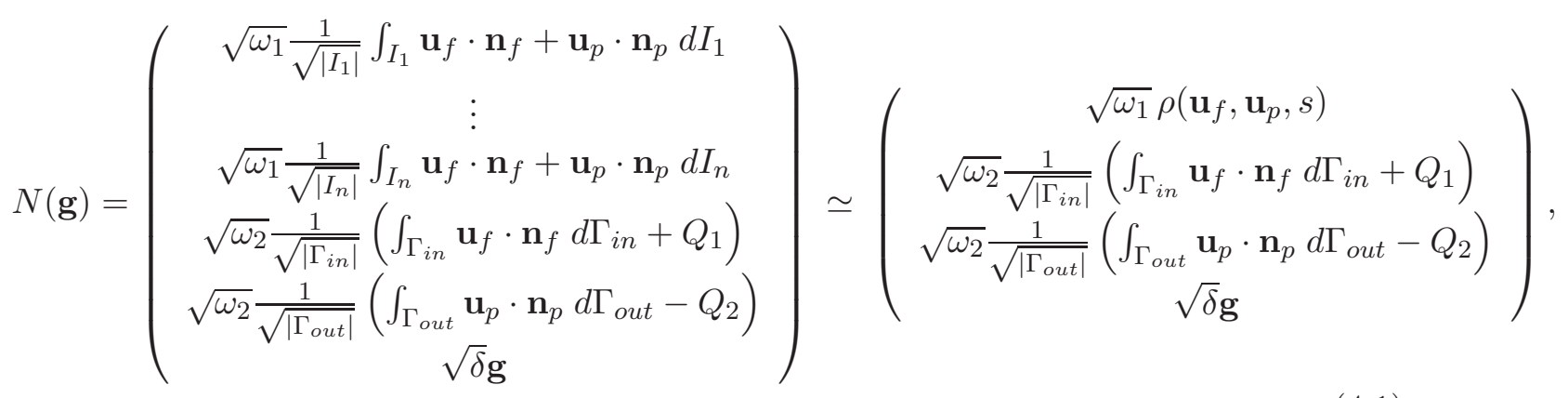

where, for $q_{i}=\int_{I_{i}} \mathbf{u}_{f} \cdot \mathbf{n}_{f}+\mathbf{u}_{p} \cdot \mathbf{n}_{p} d I_{i}, 1_{I_{i}}(s)$ the characteristic function on $I_{i}$,

$$
\rho(s)=\sum_{i=1}^{n} \frac{1}{\sqrt{\left|I_{i}\right|}} q_{i} 1_{I_{i}}(s) .
$$

$\rho(s)$ as defined in (4.2) is contained in $H^{1 / 2-\epsilon}(I)$. In the approximation scheme below $\rho(s)$ appears as an input on the right hand side of a Darcy equation as part of a well defined linear functional. For the associated continuous variational formulation, formally we require such functions to lie in $H^{1 / 2}\left(\partial \Omega_{p}\right)$. This is readily obtained by smoothing $\rho(s)$ and imposing a value of 0 for the smoothed function at the endpoints of $I$. For example, in place of $\rho(s)$, one could consider

$$
\begin{aligned}
& \rho_{s m}(s) \in Q I:=\left\{g \in C^{1}(I): g \in P_{2}\left(I_{i}\right), g=0 \text { at the endpoints of } I\right\} \\
& \text { satisfying } \int_{I_{i}} \rho_{s m}(s) d I_{i}=q_{i}, i=1,2, \ldots, n .
\end{aligned}
$$

Note that $\rho_{s m}$ defines a bounded linear mapping from $\mathbb{R}^{n}$ onto $Q I \subset L^{2}(I)$, with a bounded inverse. Hence $Q I^{-1}=Q I$.

For generality, we consider

$$
\rho(s)=\rho\left(\mathbf{u}_{f}, \mathbf{u}_{p} ; s\right)=\rho\left(q_{1}, q_{2}, \ldots, q_{n} ; s\right): \mathbb{R}^{n} \longrightarrow R I \subset L^{2}(I),
$$


to denote a bounded linear onto mapping, with a bounded inverse.

The least squares problem we consider is

$$
\min _{\mathbf{g} \in \mathbf{S}} \mathcal{J}(\mathbf{g})=\frac{1}{2} \min _{\mathbf{g} \in \mathbf{S}}\|N(\mathbf{g})\|_{R I \times R^{2} \times \mathbf{S}}^{2},
$$

which is equivalent to (3.1).

We solve this problem by a residual updating technique. First, an initial guess for a

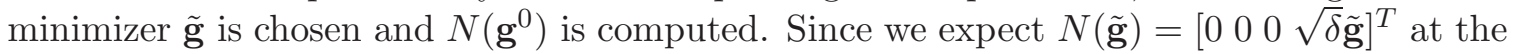
minimizer, take $N\left(\mathbf{g}^{0}\right)-\left[\begin{array}{llll}0 & 0 & 0 & \sqrt{\delta} \mathbf{g}^{0}\end{array}\right]^{T}$ as a residual and find a correction $\mathbf{h}^{0}$ for $\mathbf{g}^{0}$ such that

$$
\begin{aligned}
& \frac{1}{2}\left\|\left(N\left(\mathbf{g}^{0}\right)-\left[\begin{array}{llll}
0 & 0 & 0 & \sqrt{\delta} \mathbf{g}^{0}
\end{array}\right]^{T}\right)+N^{\prime}\left(\mathbf{g}^{0}\right)\left(\mathbf{h}^{0}\right)\right\|_{R I \times R^{2} \times \mathbf{S}}^{2}= \\
& \min _{\mathbf{h} \in \mathbf{S}} \frac{1}{2}\left\|\left(N\left(\mathbf{g}^{0}\right)-\left[\begin{array}{llll}
0 & 0 & 0 & \sqrt{\delta} \mathbf{g}^{0}
\end{array}\right]^{T}\right)+N^{\prime}\left(\mathbf{g}^{0}\right)(\mathbf{h})\right\|_{R I \times R^{2} \times \mathbf{S}}^{2} .
\end{aligned}
$$

In (4.6) $N^{\prime}\left(\mathbf{g}^{0}\right)(\cdot): \mathbf{S} \rightarrow R I \times R^{2} \times \mathbf{S}$ is defined by

$$
N^{\prime}\left(\mathbf{g}^{0}\right)(\mathbf{h})=\left(\begin{array}{c}
\sqrt{\omega_{1}} \rho\left(\mathbf{w}_{f}, \mathbf{w}_{p} ; s\right) \\
\sqrt{\omega_{2}} \frac{1}{\sqrt{\left|\Gamma_{\text {in }}\right|}} \int_{\Gamma_{\text {in }}} \mathbf{w}_{f} \cdot \mathbf{n}_{f} d \Gamma_{\text {in }} \\
\sqrt{\omega_{2}} \frac{1}{\sqrt{\left|\Gamma_{\text {out }}\right|}} \int_{\Gamma_{\text {out }}} \mathbf{w}_{p} \cdot \mathbf{n}_{p} d \Gamma_{\text {out }} \\
\sqrt{\delta} \mathbf{h}
\end{array}\right),
$$

where $\left(\mathbf{w}_{f}, \xi_{f}\right)$ and $\left(\mathbf{w}_{p}, \xi_{p}\right)$ are the solutions of

$$
\begin{aligned}
2 \nu_{f}\left(D\left(\mathbf{w}_{f}\right), D\left(\mathbf{v}_{f}\right)\right)- & \left(\xi_{f}, \nabla \cdot \mathbf{v}_{f}\right)+\alpha\left(\mathbf{w}_{f} \cdot \mathbf{t}, \mathbf{v}_{f} \cdot \mathbf{t}\right)_{I} \\
& =\left\langle h_{1}, \mathbf{v}_{f} \cdot \mathbf{n}_{f}\right\rangle_{I}+\left\langle\mathbf{h}_{2}, \mathbf{v}_{f}\right\rangle_{\Gamma_{i n}} \quad \forall \mathbf{v}_{f} \in \mathbf{X}_{f}, \\
\left(q_{f}, \nabla \cdot \mathbf{w}_{f}\right)=0 \quad \forall q_{f} & \in Q_{f},
\end{aligned}
$$

and

$$
\begin{aligned}
& \nu_{p}\left(\mathbf{w}_{p}, \mathbf{v}_{p}\right)-\left(\xi_{p}, \nabla \cdot \mathbf{v}_{p}\right)=\left\langle h_{1}, \mathbf{v}_{p} \cdot \mathbf{n}_{p}\right\rangle_{I}+\left\langle h_{3}, \mathbf{v}_{p} \cdot \mathbf{n}_{p}\right\rangle_{\Gamma_{\text {out }}} \quad \forall \mathbf{v}_{p} \in \mathbf{X}_{p} \\
& \left(q_{p}, \nabla \cdot \mathbf{w}_{p}\right)=0 \quad \forall q_{p} \in Q_{p} .
\end{aligned}
$$
$\mathbf{S}^{*}$.

Recall the adjoint operator of $N^{\prime}\left(\mathbf{g}^{0}\right)(\cdot), N^{\prime}\left(\mathbf{g}^{0}\right)^{*}(\cdot)$, satisfies $N^{\prime}\left(\mathbf{g}^{0}\right)^{*}(\cdot): R I \times R^{2} \times \mathbf{S}^{*} \rightarrow$

The following theorem allows us to use the normal equation to solve the least squares problem (4.6).

Theorem 4.1 The operator $N^{\prime}\left(\mathrm{g}^{0}\right)(\cdot)$ has a closed range. Hence, the solution of the linear least squares problem (4.6) can be obtained by solving the normal equation

$$
N^{\prime}\left(\mathbf{g}^{0}\right)^{*} N^{\prime}\left(\mathbf{g}^{0}\right)\left(\mathbf{h}^{0}\right)=-N^{\prime}\left(\mathbf{g}^{0}\right)^{*}\left(N\left(\mathbf{g}^{0}\right)-\left[\begin{array}{lllll}
0 & 0 & 0 & \sqrt{\delta} \mathbf{g}^{0}
\end{array}\right]^{T}\right) .
$$


Proof: Let $\left\{\left[\rho\left(a_{1}^{k}, a_{2}^{k}, \ldots, a_{n}^{k} ; s\right), \mathbf{b}^{k}, \mathbf{c}^{k}\right]^{T}\right\} \subset \operatorname{Range}\left(N^{\prime}\left(\mathbf{g}^{0}\right)\right)$ converge to $\left[\rho\left(\hat{a}_{1}, \hat{a}_{2}, \ldots, \hat{a}_{n} ; s\right), \widehat{\mathbf{b}}, \widehat{\mathbf{c}}\right]^{T}$. Then, there exist $\mathbf{w}_{f}^{k} \in \mathbf{X}_{f}, \mathbf{w}_{p}^{k} \in \mathbf{X}_{p}$ and $\mathbf{h}^{k} \in \mathbf{S}$ such that

$$
\begin{aligned}
a_{i}^{k} & =\sqrt{\omega_{1}} \frac{1}{\sqrt{\left|I_{i}\right|}} \int_{I_{i}} \mathbf{w}_{f}^{k} \cdot \mathbf{n}_{f}+\mathbf{w}_{p}^{k} \cdot \mathbf{n}_{p} d I_{i}, \quad i=1,2, \ldots, n \\
\mathbf{b}^{k} & =\sqrt{\omega_{2}}\left[\frac{1}{\sqrt{\left|\Gamma_{\text {in }}\right|}} \int_{\Gamma_{\text {in }}} \mathbf{w}_{f}^{k} \cdot \mathbf{n}_{f} d \Gamma_{\text {in }}, \frac{1}{\sqrt{\left|\Gamma_{\text {out }}\right|}} \int_{\Gamma_{\text {out }}} \mathbf{w}_{p}^{k} \cdot \mathbf{n}_{f} d \Gamma_{\text {out }}\right], \\
\mathbf{c}^{k} & =\sqrt{\delta} \mathbf{h}^{k}
\end{aligned}
$$

where $\left(\mathbf{w}_{f}^{k}, \xi_{f}^{k}\right)$ and $\left(\mathbf{w}_{p}^{k}, \xi_{p}^{k}\right)$ are the solutions of (4.8)-(4.11) with $\mathbf{h}$ replaced by $\mathbf{h}^{k}$. Clearly, $\mathbf{h}^{k}=\frac{\mathbf{c}^{k}}{\sqrt{\delta}} \rightarrow \frac{\widehat{\mathbf{c}}}{\sqrt{\delta}}$ and, by passing to the limit, we have that $\widehat{a}_{i}=\sqrt{\omega_{1}} \frac{1}{\sqrt{\left|I_{i}\right|}} \int_{I_{i}} \widehat{\mathbf{w}}_{f} \cdot \mathbf{n}_{f}+\widehat{\mathbf{w}}_{p}$. $\mathbf{n}_{p} d I_{i}$ for $i=1, \ldots, n$, where $\left(\widehat{\mathbf{w}}_{f}, \widehat{\xi}_{f}\right)$ and $\left(\widehat{\mathbf{w}}_{p}, \widehat{\xi}_{p}\right)$ satisfy $(4.8)-(4.11)$ with $\mathbf{h}$ replaced by $\frac{\widehat{\mathbf{c}}}{\sqrt{\delta}}$.

Hence, $N^{\prime}\left(\mathbf{g}^{0}\right)(\widehat{\mathbf{c}} / \sqrt{\delta})=\left[\rho\left(\hat{a}_{1}, \hat{a}_{2}, \ldots, \hat{a}_{n} ; s\right), \widehat{\mathbf{b}}, \widehat{\mathbf{c}}\right]^{T}$, and $N^{\prime}\left(\mathbf{g}^{0}\right)(\cdot)$ has a closed range. That $\mathbf{h}^{0}$ satisfies (4.12) follows from [15, Thm. 2.1.1].

Next we identify $N^{\prime}\left(\mathbf{g}^{0}\right)^{*}(\cdot)$ and verify that it is the adjoint operator of $N^{\prime}\left(\mathbf{g}^{0}\right)(\cdot)$.

Lemma 4.2 For $(\boldsymbol{\gamma}, \boldsymbol{\sigma}, \mathbf{y}) \in R I \times R^{2} \times \mathbf{S}^{*}$, we have

$$
N^{\prime}\left(\mathbf{g}^{0}\right)^{*}\left(\begin{array}{c}
\gamma \\
\boldsymbol{\sigma} \\
\mathbf{y}
\end{array}\right)=\left(\begin{array}{c}
\left.\left(\mathbf{z}_{f} \cdot \mathbf{n}_{f}+\mathbf{z}_{p} \cdot \mathbf{n}_{p}\right)\right|_{I} \\
\left.\mathbf{z}_{f}\right|_{\Gamma_{\text {in }}} \\
\left.\left(\mathbf{z}_{p} \cdot \mathbf{n}_{p}\right)\right|_{\Gamma_{\text {out }}}
\end{array}\right)+\sqrt{\delta} \mathbf{y}
$$

where $\left(\mathbf{z}_{f}, \varphi_{f}\right)$ and $\left(\mathbf{z}_{p}, \varphi_{p}\right)$ are the solutions of

$$
\begin{aligned}
& 2 \nu_{f}\left(D\left(\mathbf{z}_{f}\right), D\left(\mathbf{v}_{f}\right)\right)-\left(\varphi_{f}, \nabla \cdot \mathbf{v}_{f}\right)+\alpha\left(\mathbf{z}_{f} \cdot \mathbf{t}, \mathbf{v}_{f} \cdot \mathbf{t}\right)_{I} \\
& \quad=\sqrt{\omega_{1}} \int_{I} \gamma(s) \rho\left(\mathbf{v}_{f}, \mathbf{0} ; s\right) d I+\sqrt{\omega_{2}} \sigma_{1} \frac{1}{\sqrt{\left|\Gamma_{i n}\right|}} \int_{\Gamma_{i n}} \mathbf{v}_{f} \cdot \mathbf{n}_{f} d \Gamma_{i n} \quad \forall \mathbf{v}_{f} \in \mathbf{X}_{f}, \\
& \left(q_{f}, \nabla \cdot \mathbf{z}_{f}\right)=0 \quad \forall q_{f} \in Q_{f},
\end{aligned}
$$

and

$$
\begin{aligned}
& \nu_{p}\left(\mathbf{z}_{p}, \mathbf{v}_{p}\right)-\left(\varphi_{p}, \nabla \cdot \mathbf{v}_{p}\right) \\
& \quad=\sqrt{\omega_{1}} \int_{I} \gamma(s) \rho\left(\mathbf{0}, \mathbf{v}_{p} ; s\right) d \Gamma+\sqrt{\omega_{2}} \sigma_{2} \frac{1}{\sqrt{\left|\Gamma_{\text {out }}\right|}} \int_{\Gamma_{\text {out }}} \mathbf{v}_{p} \cdot \mathbf{n}_{p} d \Gamma_{\text {out }} \quad \forall \mathbf{v}_{p} \in \mathbf{X}_{p}, \\
& \left(q_{p}, \nabla \cdot \mathbf{z}_{p}\right)=0 \quad \forall q_{p} \in Q_{p} .
\end{aligned}
$$

Proof: Letting $\left(\mathbf{v}_{f}, q_{f}\right)=\left(\mathbf{z}_{f}, \varphi_{f}\right),\left(\mathbf{v}_{p}, q_{p}\right)=\left(\mathbf{z}_{p}, \varphi_{p}\right)$ in (4.8)-(4.11) and $\left(\mathbf{v}_{f}, q_{f}\right)=$ 


$$
\begin{aligned}
\left(\mathbf{w}_{f}, \xi_{f}\right),\left(\mathbf{v}_{p}, q_{p}\right)= & \left(\mathbf{w}_{p}, \xi_{p}\right) \text { in }(4.15)-(4.18), \text { we obtain } \\
\left\langle h_{1}, \mathbf{z}_{f} \cdot \mathbf{n}_{f}+\mathbf{z}_{p} \cdot\right. & \left.\mathbf{n}_{p}\right\rangle_{I}+\left\langle\mathbf{h}_{2}, \mathbf{z}_{f}\right\rangle_{\Gamma_{\text {in }}}+\left\langle h_{3}, \mathbf{z}_{p} \cdot \mathbf{n}_{p}\right\rangle_{\Gamma_{\text {out }}} \\
= & \sqrt{\omega_{1}} \int_{I} \gamma(s) \rho\left(\mathbf{v}_{f}, \mathbf{v}_{p} ; s\right) d I \\
& +\sqrt{\omega_{2}}\left[\sigma_{1} \frac{1}{\sqrt{\left|\Gamma_{\text {in }}\right|}} \int_{\Gamma_{\text {in }}} \mathbf{w}_{f} \cdot \mathbf{n}_{f} d \Gamma_{\text {in }}+\sigma_{2} \frac{1}{\sqrt{\left|\Gamma_{\text {out }}\right|}} \int_{\Gamma_{\text {out }}} \mathbf{w}_{p} \cdot \mathbf{n}_{p} d \Gamma_{\text {out }}\right] .
\end{aligned}
$$

Hence, by (4.7), (4.14) and (4.19), for $\mathbf{h}=\left(h_{1}, \mathbf{h}_{2}, h_{3}\right) \in \mathbf{S}$

$$
\begin{aligned}
\left(N^{\prime}\left(\mathbf{g}^{0}\right) \mathbf{h},\left[\begin{array}{c}
\gamma \\
\boldsymbol{\sigma} \\
\mathbf{y}
\end{array}\right]\right) & =\sqrt{\omega_{1}} \int_{I} \gamma(s) \rho\left(\mathbf{v}_{f}, \mathbf{v}_{p} ; s\right) d I+\sqrt{\omega_{2}}\left(\sigma_{1} \frac{1}{\sqrt{\left|\Gamma_{\text {in }}\right|}} \int_{\Gamma_{\text {in }}} \mathbf{w}_{f} \cdot \mathbf{n}_{f} d \Gamma_{\text {in }}\right. \\
& \left.+\sigma_{2} \frac{1}{\sqrt{\left|\Gamma_{\text {out }}\right|}} \int_{\Gamma_{\text {out }}} \mathbf{w}_{p} \cdot \mathbf{n}_{p} d \Gamma_{\text {out }}\right)+\sqrt{\delta}\langle\mathbf{h}, \mathbf{y}\rangle_{S^{*}, S} \\
& =\left\langle h_{1}, \mathbf{z}_{f} \cdot \mathbf{n}_{f}+\mathbf{z}_{p} \cdot \mathbf{n}_{p}\right\rangle_{I}+\left\langle\mathbf{h}_{2}, \mathbf{z}_{f}\right\rangle_{\Gamma_{\text {in }}}+\left\langle h_{3}, \mathbf{z}_{p} \cdot \mathbf{n}_{p}\right\rangle_{\Gamma_{\text {out }}}+\sqrt{\delta}\langle\mathbf{h}, \mathbf{y}\rangle_{S^{*}, S} \\
& =\left(\mathbf{h}, N^{\prime}\left(\mathbf{g}^{0}\right)^{*}\left[\begin{array}{c}
\gamma \\
\boldsymbol{\sigma} \\
\mathbf{y}
\end{array}\right]\right) .
\end{aligned}
$$

We adopt the following basic conjugate gradient algorithm for the linear least squares problem (4.5), which can be found in many references. For example, see [14] or [15].

Algorithm 4.3 (Conjugate Gradient Method for the Least Squares Problem)

Given $A, b$, and $x^{(0)}$,

$$
\begin{aligned}
& \text { 1. Set } r^{(0)}=b-A x^{(0)}, \\
& \quad p^{(0)}=A^{*} r^{(0)} \text {. } \\
& \text { 2. For } n=0,1,2, \cdots, \\
& \text { a. } \text { if }\left\|A^{*} r^{(n)}\right\|<\epsilon \text { stop, } \\
& \text { b. } \sigma^{(n)}=\left\|A^{*} r^{(n)}\right\|^{2} /\left\|A p^{(n)}\right\|^{2}, \\
& \text { c. } x^{(n+1)}=x^{(n)}+\sigma^{(n)} p^{(n)}, \\
& \text { d. } r^{(n+1)}=r^{(n)}-\sigma^{(n)} A p^{(n)}, \\
& \text { e. } \tau^{(n)}=\left\|A^{*} r^{(n+1)}\right\|^{2} /\left\|A^{*} r^{(n)}\right\|^{2}, \\
& \text { f. } p^{(n+1)}=A^{*} r^{(n+1)}+\tau^{(n)} p^{(n)} .
\end{aligned}
$$

Thus, the linear least squares problem (4.5) can be solved using the following residual updating algorithm.

\section{Algorithm 4.4}


1. Choose $\mathbf{g}^{0}$.

2. With $x^{(0)}=\tilde{\mathbf{h}}$, an initial guess for $\mathbf{h}$, apply Algorithm 4.3, with $A=N^{\prime}\left(\mathbf{g}^{0}\right)$, $b=-\left(N\left(\mathbf{g}^{0}\right)-\left[\begin{array}{llll}0 & 0 & 0 & \sqrt{\delta} \mathbf{g}^{0}\end{array}\right]^{T}\right)$, to determine the solution $x=\mathbf{h} ;$

3. Solution $\tilde{\mathbf{g}}=\mathbf{g}^{0}+\mathbf{h}$,

\section{Extension to a nonlinear problem}

We consider the nonlinear Stokes-Darcy problem

$$
\begin{aligned}
&-\nu_{f}\left(\left|D\left(\mathbf{u}_{f}\right)\right|\right) \nabla \cdot D\left(\mathbf{u}_{f}\right)+\nabla p_{f}=\mathbf{f}_{f} \quad \text { in } \Omega_{f}, \\
& \nabla \cdot \mathbf{u}_{f}=0 \quad \text { in } \Omega_{f}, \mathbf{u}_{f}=\mathbf{0} \quad \text { on } \Gamma_{f}, \\
&-\int_{\Gamma_{\text {in }}} \mathbf{u}_{f} \cdot \mathbf{n}_{f} d \Gamma_{f}=Q_{1}, \\
& \nu_{p}\left(\left|\mathbf{u}_{p}\right|\right) \mathbf{u}_{p}+\nabla p_{p}=\mathbf{0} \quad \text { in } \Omega_{p}, \\
& \nabla \cdot \mathbf{u}_{p}=f_{p} \quad \text { in } \Omega_{p}, \\
& \mathbf{u}_{p} \cdot \mathbf{n}_{p}=0 \quad \text { on } \Gamma_{p}, \\
& \int_{\Gamma_{\text {out }}} \mathbf{u}_{p} \cdot \mathbf{n}_{p} d \Gamma_{p}=Q_{2},
\end{aligned}
$$

where $\nu_{f}\left(\left|D\left(\mathbf{u}_{f}\right)\right|\right), \nu_{p}\left(\left|\mathbf{u}_{p}\right|\right)$ are given by the Cross model

$$
\begin{gathered}
\nu_{f}\left(\left|D\left(\mathbf{u}_{f}\right)\right|\right)=\nu_{f_{\infty}}+\frac{\nu_{f_{0}}-\nu_{f_{\infty}}}{1+K_{f}\left|D\left(\mathbf{u}_{f}\right)\right|^{2-r_{f}}}, \\
\nu_{p}\left(\left|\mathbf{u}_{p}\right|\right)=\nu_{p_{\infty}}+\frac{\nu_{p_{0}}-\nu_{p_{\infty}}}{1+K_{p}\left|\mathbf{u}_{p}\right|^{2-r_{p}}}
\end{gathered}
$$

for $r_{f}, r_{p}>1$, respectively. Note that with the choice of $r_{f}=r_{p}=2$, the system is equivalent to the linear problem (2.1)-(2.8).

The nonlinear operator $N(\mathbf{g})$ is defined as in (4.1), where $\mathbf{u}_{f}, \mathbf{u}_{p}$ satisfy

$$
\begin{aligned}
& \left(\nu_{f}\left(\left|D\left(\mathbf{u}_{f}\right)\right|\right) D\left(\mathbf{u}_{f}\right), D\left(\mathbf{v}_{f}\right)\right)-\left(p_{f}, \nabla \cdot \mathbf{v}_{f}\right)+\alpha\left(\mathbf{u}_{f} \cdot \mathbf{t}, \mathbf{v}_{f} \cdot \mathbf{t}\right)_{I} \\
& =\left(\mathbf{f}, \mathbf{v}_{f}\right)+\left\langle g_{1}, \mathbf{v}_{f} \cdot \mathbf{n}_{f}\right\rangle_{I}+\left\langle\mathbf{g}_{2}, \mathbf{v}_{f}\right\rangle_{\Gamma_{i n}} \quad \forall \mathbf{v}_{f} \in \mathbf{X}_{f}, \\
& \left(q_{f}, \nabla \cdot \mathbf{u}_{f}\right)=0 \quad \forall q_{f} \in Q_{f}, \\
& \left(\nu_{p}\left(\left|\mathbf{u}_{p}\right|\right) \mathbf{u}_{p}, \mathbf{v}_{p}\right)-\left(p_{p}, \nabla \cdot \mathbf{v}_{p}\right)=\left\langle g_{1}, \mathbf{v}_{p} \cdot \mathbf{n}_{p}\right\rangle_{I}+\left\langle g_{3}, \mathbf{v}_{p} \cdot \mathbf{n}_{p}\right\rangle_{\Gamma_{\text {out }}} \quad \forall \mathbf{v}_{p} \in \mathbf{X}_{p}, \\
& \left(q_{p}, \nabla \cdot \mathbf{u}_{p}\right)=\left(f_{p}, q_{p}\right) \quad \forall q_{p} \in Q_{p} .
\end{aligned}
$$


The linear operator $N^{\prime}\left(\mathbf{g}^{0}\right)(\mathbf{h})$ is then defined as in $(4.7)$ where $\left(\mathbf{w}_{f}, \xi_{f}\right)$ and $\left(\mathbf{w}_{p}, \xi_{p}\right)$ are the solutions of

$$
\begin{aligned}
& \left(\nu_{f}\left(\left|D\left(\mathbf{u}_{f}\right)\right|\right) D\left(\mathbf{w}_{f}\right), D\left(\mathbf{v}_{f}\right)\right) \\
& +\left(\frac{\left(r_{f}-2\right)\left(\nu_{f_{0}}-\nu_{f_{\infty}}\right) K_{f}}{\left(1+K_{f}\left|D\left(\mathbf{u}_{f}\right)\right|^{2-r_{f}}\right)^{2}\left|D\left(\mathbf{u}_{f}\right)\right|^{r_{f}}} D\left(\mathbf{u}_{f}\right)\left(D\left(\mathbf{u}_{f}\right): D\left(\mathbf{w}_{f}\right)\right), D\left(\mathbf{v}_{f}\right)\right)-\left(\xi_{f}, \nabla \cdot \mathbf{v}_{f}\right) \\
& +\alpha\left(\mathbf{w}_{f} \cdot \mathbf{t}, \mathbf{v}_{f} \cdot \mathbf{t}\right)_{I}=\left\langle h_{1}, \mathbf{v}_{f} \cdot \mathbf{n}_{f}\right\rangle_{I}+\left\langle\mathbf{h}_{2}, \mathbf{v}_{f}\right\rangle_{\Gamma_{i n}} \quad \forall \mathbf{v}_{f} \in \mathbf{X}_{f} \\
& \left(q_{f}, \nabla \cdot \mathbf{w}_{f}\right)=0 \quad \forall q_{f} \in Q_{f}
\end{aligned}
$$

and

$$
\begin{gathered}
\left(\nu_{p}\left(\left|\mathbf{u}_{p}\right|\right) \mathbf{w}_{p}, \mathbf{v}_{p}\right)+\left(\frac{\left(r_{p}-2\right)\left(\nu_{p_{0}}-\nu_{p_{\infty}}\right) K_{p}}{\left(1+K_{p}\left|\mathbf{u}_{p}\right|^{2-r_{p}}\right)^{2}\left|\mathbf{u}_{p}\right|^{r_{p}}} \mathbf{u}_{p},\left(\mathbf{u}_{p}: \mathbf{w}_{p}\right) \mathbf{v}_{p}\right)-\left(\xi_{p}, \nabla \cdot \mathbf{v}_{p}\right) \\
=\left\langle h_{1}, \mathbf{v}_{p} \cdot \mathbf{n}_{p}\right\rangle_{I}+\left\langle h_{3}, \mathbf{v}_{p} \cdot \mathbf{n}_{p}\right\rangle_{\Gamma_{\text {out }}} \quad \forall \mathbf{v}_{p} \in \mathbf{X}_{p}, \\
\left(q_{p}, \nabla \cdot \mathbf{w}_{p}\right)=0 \quad \forall q_{p} \in Q_{p} .
\end{gathered}
$$

In (5.15) and (5.17) $\mathbf{u}_{f}, \mathbf{u}_{p}$ are solutions of (5.11)-(5.14) with $\mathbf{g}=\left[g_{1}, \mathbf{g}_{2}, g_{3}\right]^{T}$ replaced by $\mathbf{g}^{0}=\left[g_{1}^{0}, \mathbf{g}_{2}^{0}, g_{3}^{0}\right]^{T}$. Similarly, the adjoint operator $N^{\prime}\left(\mathbf{g}^{0}\right)^{*}$ is defined by $(4.14)$, where $\left(\mathbf{z}_{f}, \varphi_{f}\right)$ and $\left(\mathbf{z}_{p}, \varphi_{p}\right)$ are the solutions of

$$
\begin{aligned}
& \left(\nu_{f}\left(\left|D\left(\mathbf{u}_{f}\right)\right|\right) D\left(\mathbf{z}_{f}\right), D\left(\mathbf{v}_{f}\right)\right) \\
& +\left(\frac{\left(r_{f}-2\right)\left(\nu_{f_{0}}-\nu_{f_{\infty}}\right) K_{f}}{\left(1+K_{f}\left|D\left(\mathbf{u}_{f}\right)\right|^{2-r_{f}}\right)^{2}\left|D\left(\mathbf{u}_{f}\right)\right|^{r_{f}}} D\left(\mathbf{u}_{f}\right)\left(D\left(\mathbf{u}_{f}\right): D\left(\mathbf{z}_{f}\right)\right), D\left(\mathbf{v}_{f}\right)\right)-\left(\varphi_{f}, \nabla \cdot \mathbf{v}_{f}\right) \\
& +\alpha\left(\mathbf{w}_{f} \cdot \mathbf{t}, \mathbf{v}_{f} \cdot \mathbf{t}\right)_{I} \\
& =\sqrt{\omega_{1}} \int_{I} \gamma(s) \rho\left(\mathbf{v}_{f}, \mathbf{0} ; s\right) d I+\sqrt{\omega_{2}} \sigma_{1} \frac{1}{\sqrt{\left|\Gamma_{i n}\right|}} \int_{\Gamma_{i n}} \mathbf{v}_{f} \cdot \mathbf{n}_{f} d \Gamma_{i n} \quad \forall \mathbf{v}_{f} \in \mathbf{X}_{f} \\
& \left(q_{f}, \nabla \cdot \mathbf{z}_{f}\right)=0 \quad \forall q_{f} \in Q_{f},
\end{aligned}
$$

and

$$
\begin{aligned}
& \left(\nu_{p}\left(\left|\mathbf{u}_{p}\right|\right) \mathbf{z}_{p}, \mathbf{v}_{p}\right)+\left(\frac{\left(r_{p}-2\right)\left(\nu_{p_{0}}-\nu_{p_{\infty}}\right) K_{p}}{\left(1+K_{p}\left|\mathbf{u}_{p}\right|^{2-r_{p}}\right)^{2}\left|\mathbf{u}_{p}\right|_{p}^{r_{p}}} \mathbf{u}_{p},\left(\mathbf{u}_{p}: \mathbf{z}_{p}\right) \mathbf{v}_{p}\right)-\left(\varphi_{p}, \nabla \cdot \mathbf{v}_{p}\right) \\
& \quad=\sqrt{\omega_{1}} \int_{I} \gamma(s) \rho\left(\mathbf{0}, \mathbf{v}_{p} ; s\right) d I+\sqrt{\omega_{2}} \sigma_{2} \frac{1}{\sqrt{\left|\Gamma_{\text {out }}\right|}} \int_{\Gamma_{\text {out }}} \mathbf{v}_{p} \cdot \mathbf{n}_{p} d \Gamma_{\text {out }} \quad \forall \mathbf{v}_{p} \in \mathbf{X}_{p} \\
& \left(q_{p}, \nabla \cdot \mathbf{z}_{p}\right)=0 \quad \forall q_{p} \in Q_{p} .
\end{aligned}
$$

The Cross model, representing the viscosity functions (5.9), (5.10) is known as a strong monotone operator [7], which yields an existence theorem for the nonlinear constraint (5.11)(5.14). The existence of an optimal solution can be also obtained based on the strong monotonicity as shown in [23] for the Power law model.

As the model equations are nonlinear, the nonlinear least squares problem (4.5) can be solved using the following algorithm, which is known as the Gauss-Newton method. 


\section{Algorithm 5.1}

1. Choose $\mathbf{g}^{0}$.

2. For $n=0,1,2, \ldots$,

a. with $x^{(0)}=\tilde{\mathbf{h}}$, an initial guess for $\mathbf{h}^{n}$, apply Algorithm 4.3, with $A=N^{\prime}\left(\mathbf{g}^{n}\right)$, $b=-\left(N\left(\mathbf{g}^{n}\right)-\left[\begin{array}{llll}0 & 0 & 0 & \sqrt{\delta} \mathbf{g}^{n}\end{array}\right]^{T}\right)$, to determine the solution $x=\mathbf{h}^{n} ;$

b. set $\mathbf{g}^{n+1}=\mathbf{g}^{n}+\mathbf{h}^{n}$,

c. continue.

\section{$6 \quad$ Numerical experiments}

\subsection{Experiment 1}

We tested Algorithms 4.4, 5.1 for the coupled Stokes-Darcy system with a known exact solution. Numerical experiments were performed using a non-physical example to investigate convergence of solutions with decreasing grid sizes. The subdomains chosen were $\Omega_{f}=(0,1) \times(1,2)$ and $\Omega_{p}=(0,1) \times(0,1)$, with $I=\{(x, y): 0<x<1, y=1\}$.

Using the parameters $\nu_{f_{\infty}}=\nu_{p_{\infty}}=0.5, \nu_{f_{0}}=\nu_{p_{0}}=1, K_{f}=K_{p}=1$, the right hand side functions and boundary conditions on $\Gamma_{f} \cup \Gamma_{i n}, \Gamma_{p} \cup \Gamma_{\text {out }}$ were appropriately given so that the exact solution was

$$
\begin{gathered}
\mathbf{u}_{f}=\left[(y-1)^{2} x^{3},-e^{1} \cos (y)\right]^{T}, \quad p_{f}=\cos (x) e^{y}+y^{2}-2 y+1, \\
\mathbf{u}_{p}=\left[-x\left(\sin (y) e^{1}+2(y-1)\right),-\cos (y) e^{1}+(y-1)^{2}\right]^{T}, \quad p_{p}=-\sin (y) e^{1}+\cos (x) e^{y}+y^{2}-2 y+1 .
\end{gathered}
$$

The chosen exact solution satisfies the Beavers-Joseph-Saffmann condition (2.11) with $\alpha=$ 1. In order that the approximations converged to (6.1)-(6.2), $\mathbf{u}_{f}, \mathbf{u}_{p} \cdot \mathbf{n}$ were specified on $\Gamma_{\text {in }}$ and $\Gamma_{\text {out }}$, respectively. The Taylor-Hood $P 2-P 1$ velocity-pressure approximating elements were used for both the Stokes and the Darcy problems on structured meshes. For approximations of the Darcy problem the stabilization term $\beta\left(\nabla \cdot \mathbf{u}_{p}, \nabla \cdot \mathbf{v}_{p}\right)$ was added to (2.17) and (5.13), which ensures $\nabla \cdot \mathbf{u}_{p}^{h} \approx 0$. Consequently, the analogous terms were added to (4.10), (4.17), (5.17) and (5.21). The parameter value $\beta=100$ was used in all experiments.

First, we solved the Stokes and the Darcy problem independently using the exact boundary conditions on the interface, to compare with results by optimization. The errors using the exact boundary conditions are presented in Table 6.1 and Table 6.3 for the linear $\left(r_{f}=r_{p}=2\right)$ and the nonlinear $\left(r_{f}=f_{p}=1.5\right)$ problems, respectively.

In the objective functional (4.5) we set $\delta=10^{-10}, \omega_{1}=1, \omega_{2}=0, n=2(k-1)$, with $k$ is the number of grid points on $I$, and used $\rho(s)$ given by (4.2). As an initial control the constant function $\mathrm{g}^{0}=-0.1$ was chosen and the constant function $x^{0}=0.01$ was used to start the CG algorithm. Results for the linear and the nonlinear problems by Algorithms 4.4, 5.1 are presented in Table 6.2 and Table 6.4, respectively. For the nonlinear case, a chosen stopping criterion $\left(\mathcal{J}<10^{-7}\right)$ was met after only two iterations of the GaussNewton. In this test we observed that solutions by the optimization approach were as 
accurate as solutions obtained by using the exact boundary conditions, yielding the same rates of convergence.

Remark 6.1 The algorithms were also tested with an increased number of subsections on I. Namely, for $n=4(k-1)$ no difference in accuracy of the solutions was observed. However, with fewer subsections, $n=k-1$, the solutions lost some accuracy. We believe that the number of subsections required for optimal accuracy of a solution depends upon by several factors, such as the regularity of the true solution and the polynomial degree of the finite element spaces used.

\begin{tabular}{|c|c|c|c|c|c|c|}
\hline & \multicolumn{4}{|c|}{$\mathbf{u}_{f}$} & \multicolumn{2}{|c|}{$p_{f}$} \\
\hline grid & $L^{2}$ error & $L^{2}$ rate & $H^{1}$ error & $H^{1}$ rate & $L^{2}$ error & $L^{2}$ rate \\
\hline $3 \times 3$ & $1.24 \cdot 10^{-2}$ & & 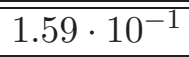 & & $2.34 \cdot 10^{-1}$ & \\
\hline $5 \times 5$ & $1.30 \cdot 10^{-3}$ & 3.26 & $3.64 \cdot 10^{-2}$ & 2.13 & $7.58 \cdot 10^{-2}$ & 1.62 \\
\hline $9 \times 9$ & $1.45 \cdot 10^{-4}$ & 3.17 & $8.74 \cdot 10^{-3}$ & 2.06 & $2.20 \cdot 10^{-2}$ & 1.78 \\
\hline $17 \times 17$ & $1.72 \cdot 10^{-5}$ & 3.07 & $2.15 \cdot 10^{-3}$ & 2.02 & $5.93 \cdot 10^{-3}$ & 1.89 \\
\hline \multirow[t]{3}{*}{$33 \times 33$} & $2.11 \cdot 10^{-6}$ & 3.03 & $5.35 \cdot 10^{-4}$ & 2.01 & $1.54 \cdot 10^{-3}$ & 1.95 \\
\hline & \multicolumn{4}{|c|}{$\mathbf{u}_{p}$} & \multicolumn{2}{|c|}{$p_{p}$} \\
\hline & $L^{2}$ error & $L^{2}$ rate & $H^{d i v}$ error & $H^{\text {div }}$ rate & $L^{2}$ error & $L^{2}$ rate \\
\hline $3 \times 3$ & $2.23 \cdot 10^{-1}$ & & $5.91 \cdot 10^{-4}$ & & $6.60 \cdot 10^{-2}$ & \\
\hline $5 \times 5$ & $6.07 \cdot 10^{-2}$ & 1.88 & $1.65 \cdot 10^{-4}$ & 1.84 & $1.59 \cdot 10^{-2}$ & 2.05 \\
\hline $9 \times 9$ & $1.65 \cdot 10^{-2}$ & 1.88 & $4.24 \cdot 10^{-5}$ & 1.96 & $3.86 \cdot 10^{-3}$ & 2.04 \\
\hline $17 \times 17$ & $4.44 \cdot 10^{-3}$ & 1.90 & $1.07 \cdot 10^{-5}$ & 1.98 & $9.56 \cdot 10^{-4}$ & 2.01 \\
\hline $33 \times 33$ & $1.18 \cdot 10^{-3}$ & 1.91 & $2.70 \cdot 10^{-6}$ & 1.99 & $2.38 \cdot 10^{-5}$ & 2.00 \\
\hline
\end{tabular}

Table 6.1: Errors using the exact boundary condition on $I$ for $r_{f}=r_{p}=2$.

\subsection{Experiment 2}

The defective boundary conditions (2.4), (2.8) were considered for the same flow domains as in Experiment 1. In (2.1)-(2.8) the right hand side functions $\mathbf{f}_{f}, f_{p}$ were chosen to be $\mathbf{0}$ and 0 , respectively. The inflow and outflow boundaries were specified as $\Gamma_{\text {in }}=\{0.75 \leq x \leq$ $1, y=2\}, \Gamma_{\text {out }}=\{0 \leq x \leq 0.25, y=0\}$, where the flow rate conditions with $Q_{1}=Q_{2}=3$ were imposed. All parameter values were chosen as in Experiment 1 and the same finite elements on the $17 \times 17$ structured mesh for each flow domain were used in all computations. The weights $\omega_{1}, \omega_{2}=1$ were chosen.

First, the control $\mathbf{g}=\left(g_{1}, \mathbf{g}_{2}, 0\right)$ was used (i.e. $\left.g_{3}=0\right)$ for the linear case $\left(r_{f}=r_{p}=2\right)$. (See the comment following (3.4)). Figure 6.1 shows the contour plot for the magnitude of the velocity and streamlines of the controlled flow in the entire flow domain. The vertical component of the computed velocity on the inflow and outflow boundaries is presented in Figure 6.2. For the nonlinear case with $r_{f}=f_{p}=1.5$ similar results were obtained.

Next we considered the control $\mathbf{g}=\left(g_{1}, \mathbf{g}_{2}, g_{3}\right)$. Figures $6.3,6.4$ show the streamlines and the vertical component of the computed velocity on the inflow and outflow boundaries. As shown in Figure 6.4, the computed solution has fluid flowing into the domain on a part 


\begin{tabular}{|c|c|c|c|c|c|c|}
\hline & \multicolumn{4}{|c|}{$\mathbf{u}_{f}$} & \multicolumn{2}{|c|}{$p_{f}$} \\
\hline grid & $L^{2}$ error & $L^{2}$ rate & $H^{1}$ error & $H^{1}$ rate & $L^{2}$ error & $L^{2}$ rate \\
\hline $3 \times 3$ & $\bar{~} 1.17 \cdot 10^{-2}$ & & $\bar{~} 1.58 \cdot 10^{-1}$ & & $2.38 \cdot 10^{-1}$ & \\
\hline $5 \times 5$ & $1.27 \cdot 10^{-3}$ & 3.20 & $3.62 \cdot 10^{-2}$ & 2.12 & $7.58 \cdot 10^{-2}$ & 1.65 \\
\hline $9 \times 9$ & $1.45 \cdot 10^{-4}$ & 3.13 & $8.72 \cdot 10^{-3}$ & 2.05 & $2.20 \cdot 10^{-2}$ & 1.79 \\
\hline $17 \times 17$ & $1.73 \cdot 10^{-5}$ & 3.07 & $2.15 \cdot 10^{-3}$ & 2.02 & $5.92 \cdot 10^{-3}$ & 1.89 \\
\hline \multirow[t]{3}{*}{$33 \times 33$} & $2.12 \cdot 10^{-6}$ & 3.03 & $5.35 \cdot 10^{-4}$ & 2.01 & $1.54 \cdot 10^{-3}$ & 1.95 \\
\hline & \multicolumn{4}{|c|}{$\overline{\mathbf{u}_{p}}$} & \multicolumn{2}{|c|}{$\overline{p_{p}}$} \\
\hline & $L^{2}$ error & $L^{2}$ rate & $H^{d i v}$ error & $H^{\text {div }}$ rate & $L^{2}$ error & $L^{2}$ rate \\
\hline$\overline{3 \times 3}$ & $\bar{~} 1.39 \cdot 10^{-1}$ & & $\bar{~} 6.61 \cdot 10^{-4}$ & & $\bar{~} 6.31 \cdot 10^{-2}$ & \\
\hline $5 \times 5$ & $3.87 \cdot 10^{-2}$ & 1.85 & $1.82 \cdot 10^{-4}$ & 1.86 & $1.56 \cdot 10^{-2}$ & 2.02 \\
\hline $9 \times 9$ & $1.09 \cdot 10^{-2}$ & 1.82 & $4.62 \cdot 10^{-5}$ & 1.98 & $3.84 \cdot 10^{-3}$ & 2.02 \\
\hline $17 \times 17$ & $3.01 \cdot 10^{-3}$ & 1.86 & $1.15 \cdot 10^{-5}$ & 2.00 & $9.55 \cdot 10^{-4}$ & 2.01 \\
\hline $33 \times 33$ & $8.04 \cdot 10^{-4}$ & 1.90 & $2.87 \cdot 10^{-6}$ & 2.00 & $2.38 \cdot 10^{-5}$ & 2.00 \\
\hline
\end{tabular}

Table 6.2: Errors using Algorithm 4.4 for $r_{f}=r_{p}=2$.

\begin{tabular}{|c|c|c|c|c|c|c|}
\hline & \multicolumn{4}{|c|}{$\mathbf{u}_{f}$} & \multicolumn{2}{|c|}{$p_{f}$} \\
\hline grid & $L^{2}$ error & $L^{2}$ rate & $H^{1}$ error & $H^{1}$ rate & $L^{2}$ error & $L^{2}$ rate \\
\hline $3 \times 3 \times 3$ & $\bar{~} 1.24 \cdot 10^{-2}$ & & $1.59 \cdot 10^{-1}$ & & $2.05 \cdot 10^{-1}$ & \\
\hline $5 \times 5$ & $1.30 \cdot 10^{-3}$ & 3.26 & $3.64 \cdot 10^{-2}$ & 2.13 & $6.63 \cdot 10^{-2}$ & 1.63 \\
\hline $9 \times 9$ & $1.45 \cdot 10^{-4}$ & 3.17 & $8.74 \cdot 10^{-3}$ & 2.06 & $1.88 \cdot 10^{-2}$ & 1.82 \\
\hline $17 \times 17$ & $1.72 \cdot 10^{-5}$ & 3.07 & $2.15 \cdot 10^{-3}$ & 2.02 & $5.02 \cdot 10^{-3}$ & 1.91 \\
\hline \multirow[t]{3}{*}{$33 \times 33$} & $2.11 \cdot 10^{-6}$ & 3.03 & $5.36 \cdot 10^{-4}$ & 2.01 & $1.29 \cdot 10^{-3}$ & 1.95 \\
\hline & \multicolumn{4}{|c|}{$\mathbf{u}_{p}$} & \multicolumn{2}{|c|}{$p_{p}$} \\
\hline & $L^{2}$ error & $L^{2}$ rate & $H^{d i v}$ error & $H^{\text {div }}$ rate & $L^{2}$ error & $L^{2}$ rate \\
\hline $3 \times 3$ & $2.44 \cdot 10^{-1}$ & & 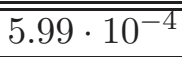 & & 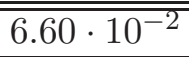 & \\
\hline $5 \times 5$ & $6.64 \cdot 10^{-2}$ & 1.88 & $1.65 \cdot 10^{-4}$ & 1.89 & $1.59 \cdot 10^{-2}$ & 2.05 \\
\hline $9 \times 9$ & $1.81 \cdot 10^{-2}$ & 1.88 & $4.23 \cdot 10^{-5}$ & 1.97 & $3.87 \cdot 10^{-3}$ & 2.04 \\
\hline $17 \times 17$ & $4.86 \cdot 10^{-3}$ & 1.90 & $1.07 \cdot 10^{-5}$ & 1.99 & $9.59 \cdot 10^{-4}$ & 2.01 \\
\hline $33 \times 33$ & $1.29 \cdot 10^{-3}$ & 1.91 & $2.68 \cdot 10^{-6}$ & 1.99 & $2.39 \cdot 10^{-4}$ & 2.00 \\
\hline
\end{tabular}

Table 6.3: Errors using the exact boundary condition on $I$ for $r_{f}=r_{p}=1.5$.

of $\Gamma_{\text {out }}$. Thus, $\Gamma_{\text {out }}$ is not in fact an outflow boundary and this causes instability for the Darcy flow near $\Gamma_{\text {out }}$.

The problem we approximated, (2.1)-(2.11), is not mathematically well-posed problem due to the defective boundary conditions. Therefore, we expect that there are many local optimal solutions to (3.4) and a different numerical solution could be obtained by using a different initial guess. 


\begin{tabular}{|c|c|c|c|c|c|c|}
\hline & \multicolumn{4}{|c|}{$\mathbf{u}_{f}$} & \multicolumn{2}{|c|}{$p_{f}$} \\
\hline grid & $L^{2}$ error & $L^{2}$ rate & $H^{1}$ error & $H^{1}$ rate & $L^{2}$ error & $L^{2}$ rate \\
\hline$\overline{3 \times 3}$ & 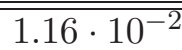 & & $\bar{~} 1.58 \cdot 10^{-1}$ & & $2.09 \cdot 10^{-1}$ & \\
\hline $5 \times 5$ & $1.27 \cdot 10^{-3}$ & 3.19 & $3.62 \cdot 10^{-2}$ & 2.12 & $6.63 \cdot 10^{-2}$ & 1.66 \\
\hline $9 \times 9$ & $1.46 \cdot 10^{-4}$ & 3.13 & $8.72 \cdot 10^{-3}$ & 2.05 & $1.88 \cdot 10^{-2}$ & 1.82 \\
\hline $17 \times 17$ & $1.73 \cdot 10^{-5}$ & 3.07 & $2.15 \cdot 10^{-3}$ & 2.02 & $5.01 \cdot 10^{-3}$ & 1.91 \\
\hline \multirow[t]{3}{*}{$33 \times 33$} & $2.11 \cdot 10^{-6}$ & 3.04 & $5.35 \cdot 10^{-4}$ & 2.01 & $1.29 \cdot 10^{-3}$ & 1.95 \\
\hline & \multicolumn{4}{|c|}{$\overline{\mathbf{u}_{p}}$} & \multicolumn{2}{|c|}{$\overline{p_{p}}$} \\
\hline & $L^{2}$ error & $L^{2}$ rate & $H^{d i v}$ error & $H^{\text {div }}$ rate & $L^{2}$ error & $L^{2}$ rate \\
\hline 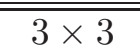 & $101.57 \cdot 10^{-1}$ & & $\bar{~} 6.64 \cdot 10^{-4}$ & & 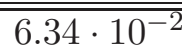 & \\
\hline $5 \times 5$ & $4.36 \cdot 10^{-2}$ & 1.85 & $1.82 \cdot 10^{-4}$ & 1.87 & $1.56 \cdot 10^{-2}$ & 2.02 \\
\hline $9 \times 9$ & $1.23 \cdot 10^{-2}$ & 1.82 & $4.61 \cdot 10^{-5}$ & 1.98 & $3.85 \cdot 10^{-3}$ & 2.02 \\
\hline $17 \times 17$ & $3.39 \cdot 10^{-3}$ & 1.86 & $1.15 \cdot 10^{-5}$ & 2.01 & $9.57 \cdot 10^{-4}$ & 2.01 \\
\hline $33 \times 33$ & $9.03 \cdot 10^{-4}$ & 1.91 & $2.86 \cdot 10^{-6}$ & 2.00 & $2.38 \cdot 10^{-5}$ & 2.00 \\
\hline
\end{tabular}

Table 6.4: Errors using Algorithm 5.1 for $r_{f}=r_{p}=1.5$.

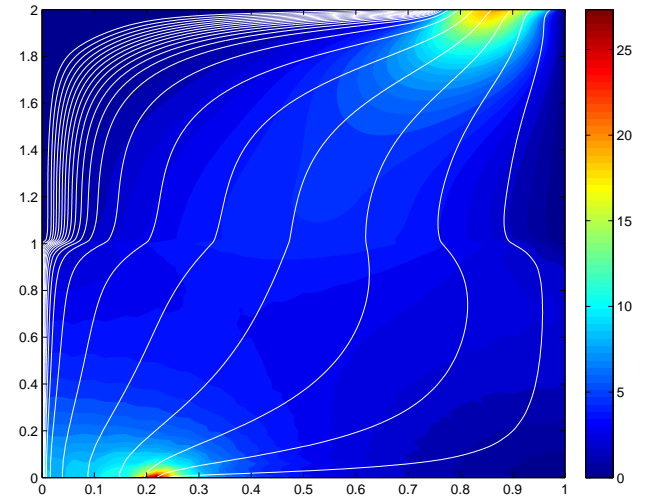

Figure 6.1: Plot of the magnitude of the velocity and streamlines by $\mathbf{g}=\left(g_{1}, \mathbf{g}_{2}, 0\right)$.
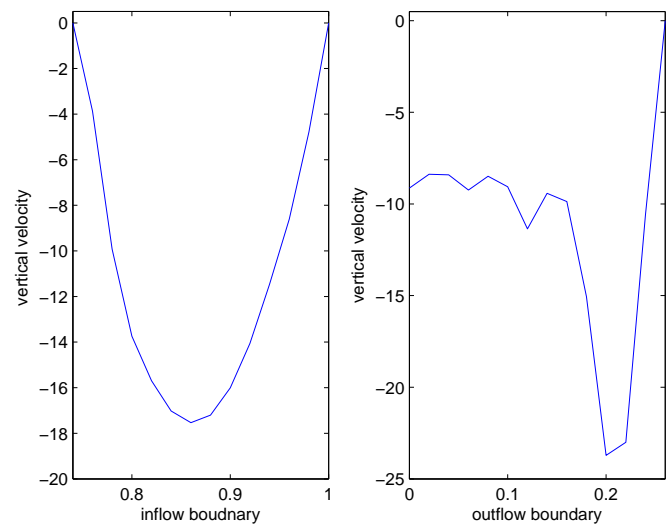

Figure 6.2: vertical velocities on inflow and outflow boundaries by $\mathbf{g}=\left(g_{1}, \mathbf{g}_{2}, 0\right)$.

\section{Conclusion}

We considered a numerical method for the linear/nonlinear Stokes-Darcy system with defective boundary conditions. The coupled problem was formulated as a minimization problem, where the system was decoupled through a control function on the interface. We showed that the proposed algorithm found an optimal control function, generating a solution for the Stokes-Darcy system satisfying both the interface conditions and the defective boundary conditions.

The method we investigated has several attractive features such as (i) decomposition of the coupled system into Stokes and Darcy subproblems (enabling existing software and solution algorithms for the Stokes and Darcy problems to be used), (ii) solution of the 


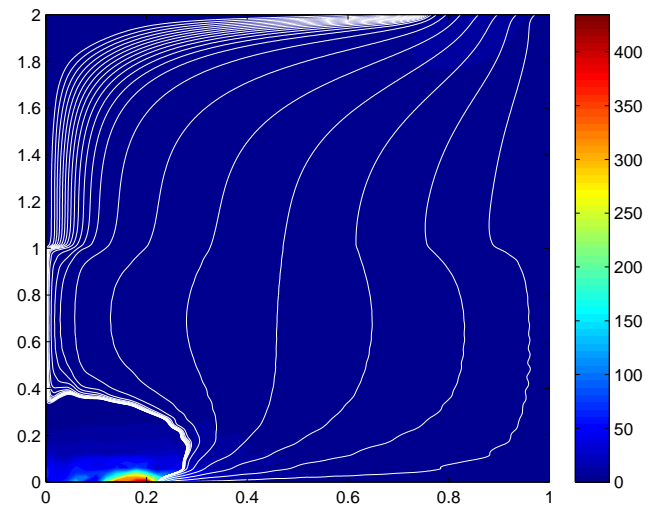

Figure 6.3: Plot of the magnitude of the velocity and streamlines by $\mathrm{g}=$ $\left(g_{1}, \mathbf{g}_{2}, g_{3}\right)$.
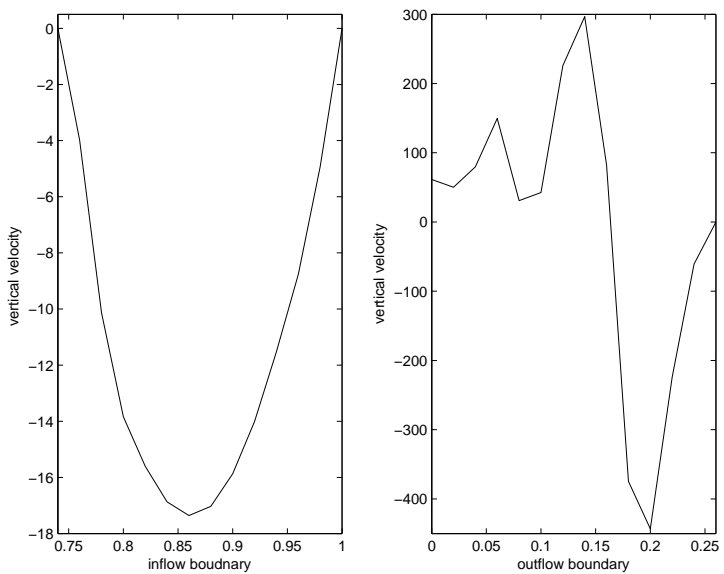

Figure 6.4: vertical velocities on inflow and outflow boundaries by $\mathbf{g}=\left(g_{1}, \mathbf{g}_{2}, g_{3}\right)$.

Stokes and Darcy subproblems in parallel, (iii) solvability of the least squares problem by the $\mathrm{CG}$ algorithm without forming the normal equation explicitly.

Another attractive feature of the method is its efficiency, in particular for the nonlinear case. It was observed in all computations that only two or three Guass-Newton iterations were required to reduce the objective functional below the specified tolerance of $10^{-8}$. In the nonlinear case the operators $N^{\prime}$ and $N^{\prime *}$ are still linear (functions of the solution to linearized Stokes and Darcy subproblems), whereas $N\left(\mathbf{g}^{n}\right)$ is a function of the (nonlinear) Stokes and Darcy subproblems. Thus, one only needs to solve decoupled nonlinear subproblems two or three times in the solution process. This is a great advantage of the method over numerical solution methods for the coupled nonlinear Stokes-Darcy system.

\section{References}

[1] T. Arbogast and D.S. Brunson. A computational method for approximating a DarcyStokes system governing a vuggy porous medium. Comput. Geosci., 11(3):207-218, 2007 .

[2] T. Arbogast and M. Gomez. A discretization and multigrid solver for a Darcy-Stokes system governing a vuggy porous medium. Comput. Geosci., 13(3):331-348, 2009.

[3] C. Bernardi, T.C. Rebollo, F. Hecht, and Z. Mghazli. Mortar finite element discretization of a model coupling Darcy and Stokes equations. Math. Model. Numer. Anal., 42(3):375-410, 2008.

[4] M. Cai, M. Mu, and J. Xu. Numerical solution to a mixed Navier-Stokes/Darcy model by the two-grid approach. SIAM J. Numer. Anal., 47:3325-3338, 2009. 
[5] Y. Cao, M. Gunzburger, X. He, and X. Wang. Robin-Robin domain decomposition methods for the steady-state Stokes-Darcy system with the Beavers-Joseph interface condition. Numer. Math., 117:601-629, 2011.

[6] A. Cesmelioglu and B. Riviére. Primal discontinuous Galerkin methods for timedependent coupled surface and subsurface flow. J. Sci. Comput., 40:115-140, 2009.

[7] S.-S. Chow and G.F. Carey. Numerical approximation of generalized Newtonian fluids using Powell-Sabin-Heindl elements: I. Theoretical estimates. Int. J. Numer. Meth. Fluids, 41:1085-1118, 2003.

[8] M. Discacciati, E. Miglio, and A. Quarteroni. Mathematical and numerical models for coupling surface and groundwater flows. Appl. Numer. Math., 43:57-74, 2001.

[9] M. Discacciati, A. Quarteroni, and A. Vali. Robin-Robin domain decomposition methods for the Stokes-Darcy coupling. SIAM J. Numer. Anal., 45:1246-1268, 2007.

[10] V.J. Ervin, E.W. Jenkins, and S. Sun. Coupled generalized nonlinear Stokes flow with flow through a porous medium. SIAM J. Numer. Anal., 47:929-952, 2009.

[11] V.J. Ervin, E.W. Jenkins, and S. Sun. Coupling non-linear Stokes and Darcy flow using mortar finite elements. Appl. Numer. Math., 61:1198-1222, 2011.

[12] J. Galvis and M. Sarkis. Non-matching mortar discretization analysis for the coupling Stokes-Darcy equations. Electron. Trans. Numer. Anal., 26:350-384, 2007.

[13] J. Galvis and M. Sarkis. FETI and BDD preconditioners for Stokes-mortar-Darcy systems. Commun. Appl. Math. Comput. Sci., 5:1-30, 2010.

[14] G. Golub and C. van Loan. Matrix Computations. Johns Hopkins University, Baltimore, 1989.

[15] C.W. Groetsch. Generalized Inverses of Linear Operators. Marcel Dekker, New York, 1977.

[16] M.D. Gunzburger and H. Lee. An optimization-based domain decomposition method for the Navier-Stokes equations. SIAM J. Numer. Anal., 37:1455-1480, 2000.

[17] M.D. Gunzburger, J.S. Peterson, and H. Kwon. An optimization based domain decomposition method for partial differential equations. Comput. Math. Appl., 37:77-93, 1999.

[18] N.S. Hanspal, A.N. Waghode, V. Nassehi, and R.J. Wakeman. Numerical analysis of coupled Stokes/Darcy flows in industrial filtrations. Transp. Porous Media, 64:15731634, 2006.

[19] J.G. Heywood, R. Rannacher, and S. Turek. Artificial boundaries and flux and pressure conditions for the incompressible Navier-Stokes equations. Int. J. Numer. Methods Fluids, 22:325-352, 1996. 
[20] W. Jager and A. Mikelić. On the interface boundary condition of Beaver, Joseph and Saffman. SIAM J. Appl. Math., 60:1111-1127, 2000.

[21] W.J. Layton, F. Schieweck, and I. Yotov. Coupling fluid flow with porous media flow. SIAM J. Numer. Anal., 40:2195-2218, 2003.

[22] H. Lee. An optimization-based domain decomposition method for the Boussinesq equations. Numer. Methods Partial Differential Equations, 18:1-25, 2002.

[23] H. Lee. Optimal control for quasi-Newtonian flows with defective boundary conditions. Comput. Methods. Appl. Mech. Engrg., 200:2498-2506, 2011.

[24] B. Riviére. Analysis of a discontinuous finite element method for the coupled Stokes and Darcy problems. J. Sci. Comput., 22(23):479-500, 2005.

[25] B. Riviére and I. Yotov. Locally conservative coupling of Stokes and Darcy flows. SIAM J. Numer. Anal., 42(5):1959-1977, 2005. 